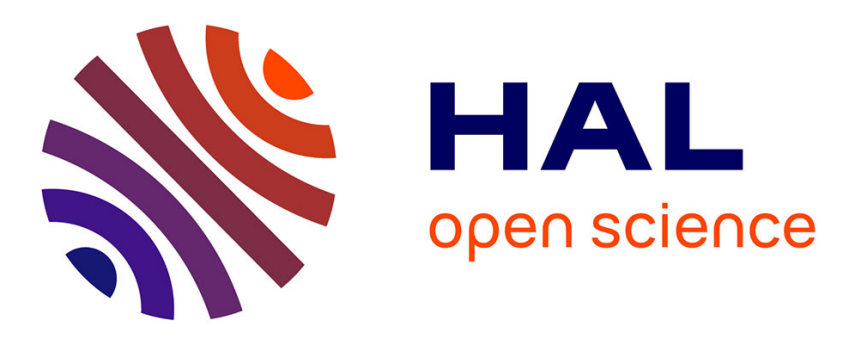

\title{
Hybrid PID control for transient performance improvement of motion systems with friction
}

R. Beerens, Andrea Bisoffi, Luca Zaccarian, W.P.M.H. P M H Heemels, H. Nijmeijer, N. van de Wouw

\section{- To cite this version:}

R. Beerens, Andrea Bisoffi, Luca Zaccarian, W.P.M.H. P M H Heemels, H. Nijmeijer, et al.. Hybrid PID control for transient performance improvement of motion systems with friction. American Control Conference (ACC 2018), Jun 2018, Milwaukee (WI), United States. pp.539-544, 10.23919/ACC.2018.8431613 . hal-01970887

\section{HAL Id: hal-01970887 \\ https: / hal.laas.fr/hal-01970887}

Submitted on 6 Jan 2019

HAL is a multi-disciplinary open access archive for the deposit and dissemination of scientific research documents, whether they are published or not. The documents may come from teaching and research institutions in France or abroad, or from public or private research centers.
L'archive ouverte pluridisciplinaire HAL, est destinée au dépôt et à la diffusion de documents scientifiques de niveau recherche, publiés ou non, émanant des établissements d'enseignement et de recherche français ou étrangers, des laboratoires publics ou privés. 


\title{
Hybrid PID control for transient performance improvement of motion systems with friction ${ }^{\star}$
}

\author{
R. Beerens ${ }^{1}$, A. Bisoffi ${ }^{2}$, L. Zaccarian ${ }^{3}$, W.P.M.H. Heemels ${ }^{1}$, H. Nijmeijer ${ }^{1}$ and N. van de Wouw ${ }^{1,4}$
}

\begin{abstract}
We present a novel reset control approach to improve transient performance of a PID-controlled motion system subject to friction. In particular, a reset integrator is applied to circumvent the depletion and refilling process of a linear integrator when the system overshoots the setpoint, thereby significantly reducing settling times. Moreover, robustness for unknown static friction levels is obtained. A hybrid closed-loop system formulation is derived, and stability follows from a discontinuous Lyapunov-like function and a meagre-limsup invariance argument. The working principle of the controller is illustrated by means of a numerical example.
\end{abstract}

\section{INTRODUCTION}

In this paper, we present a reset control approach to improve transient performance of a PID-controlled mechanical motion system subject to friction. Especially in high-precision positioning systems, friction is a performance limiting factor. The presence of Coulomb friction may induce non-zero steady-state positioning errors, and the presence of the velocity-weakening (Stribeck) effect may induce so-called hunting limit cycles [1], [2], which compromises position accuracy as well.

In the past decades, many different control approaches have been developed to either compensate for friction, or to achieve high-precision positioning despite the presence of frictional effects. Friction compensation techniques make use of a parametric friction model in the control loop (see, e.g., [1], [3]-[5]) or in a calibration procedure (see, e.g., [6]). Obtaining an accurate friction model is in general a difficult task, since it is challenging to exactly capture the physics associated with friction into a model with limited complexity, suitable for online implementation. Moreover, model mismatches and a changing friction characteristic may lead to over- or undercompensation of friction [3]. Non-model-based control techniques do not aim at friction compensation, but change the effect of friction on the closed-loop system to obtain the desired performance. Examples are impulsive control [7] or dithering-based controllers [8]. A drawback of these control schemes is that the use of impulsive control forces may result in excitation of unmodeled, high-frequency system dynamics.

Although several successful applications of the above control approaches have been presented in the literature, linear (loop-shaped) controllers are still applied in the vast majority of industrial motion systems, due to the intuitive design and tuning tools for such controllers, and knowledge and experience of control practitioners. In particular, the classical PID controller is most commonly used for frictional systems, since the integrator action is capable of compensating for unknown static friction by integrating the position error. However, also PID control is prone to performance limitations. In the presence of Coulomb friction, a limitation is the slow convergence and the resulting long settling times [9]. Namely, an integrator action is required to escape a stick phase by building up the control force to overcome the (possibly unknown) static friction. However, if the position overshoots the setpoint, the integrator buffer needs to deplete and refill in order to change sign to overcome the static friction again. This process takes increasingly more time with a decreasing position error, resulting in long settling times.

In [10], a controller has been proposed to improve transient performance (besides compensating robustly for the Stribeck effect) by employing a switched PID controller, which resets the integrator state in such a way that a large part of the depletion/refilling process is circumvented. The switching mechanism relies on online identification of the static friction, which requires exact detection of stick-slip transitions. In practice, however, velocity measurement noise and discrete sampling may lead to the inability of detecting zero velocity and thus prevent such exact identification of the static friction, which may lead in turn to small steady-state limit cycling around the setpoint. In this work, we present a novel reset integrator control scheme that does not suffer from the robustness issues described above, while still significantly improving transient performance.

Reset integrators have been used to enhance transient performance of linear motion systems, see, e.g., [11]-[16] but, to the best of the authors' knowledge, not of systems with friction (which are inherently nonlinear).

The main contributions of this paper are twofold. First, we design a novel reset PID controller for systems with friction that both improves transient performance with respect to a classical PID controller, and induces robust stability with respect to uncertainties in the static friction. Second, we analyse stability of the resulting hybrid closed-loop system exploiting a meagre-limsup invariance argument [17, §8.4].

\footnotetext{
${ }^{\star}$ This research is part of the research programme High Tech Systems and Materials (HTSM), which is supported by NWO domain Applied and Engineering Sciences and partly funded by the Ministry of Economic Affairs.

${ }^{1}$ R. Beerens, M. Heemels, H. Nijmeijer, and N. van de Wouw are with the Department of Mechanical Engineering, Eindhoven University of Technology, P.O. Box 513, 5600 MB Eindhoven, The Netherlands $\{r$.beerens; w.p.m.h.heemels; h.nijmeijer; n.v.d.wouw; \}@tue.nl

${ }^{2}$ A. Bisoffi is with the Department of Automatic Control, KTH Royal Institute of Technology, 10044 Stockholm, Sweden bisoffi@kth.se

${ }^{3}$ L. Zaccarian is with CNRS, LAAS, Univ. de Toulouse, LAAS, F-31400 Toulouse, France, and also with Dip. di Ingegneria Industriale, University of Trento, 38122 Trento, Italy zaccariand laas.fr

${ }^{4} \mathrm{~N}$. van de Wouw is also with the Department of Civil, Environmental and GeoEngineering, University of Minnesota, Minneapolis, MN 55455 USA, and with the Delft Center for Systems and Control, Delft University of Technology, Delft, The Netherlands.
} 
This paper is organized as follows. In Section II, a model of the considered motion system with a classical PID controller is presented together with the reset integrator control law. The closed-loop dynamics are written in a hybrid systems formalism in Section III and a stability analysis is given in Section IV. Section V provides an illustrative example and conclusions are presented in Section VI.

Notation: $\operatorname{sign}(\cdot)($ with a lower-case $s)$ denotes the classical sign function, i.e., $\operatorname{sign}(y):=y /|y|$ for $y \neq 0$ and $\operatorname{sign}(0):=0$. $\operatorname{Sign}(\cdot)($ with an upper-case $\mathbf{S})$ denotes the set-valued $\operatorname{sign}$ function, i.e., $\operatorname{Sign}(y):=\{\operatorname{sign}(y)\}$ for $y \neq 0$, and $\operatorname{Sign}(y):=$ $[-1,1]$ for $y=0$. For $c>0$, the deadzone function is defined as: $\mathrm{dz}_{c}(x):=0$ if $|x| \leq c, \mathrm{dz}_{c}(x):=x-c$ if $x>c$, $\mathrm{dz}_{c}(x):=x+c$ if $x<-c$. A function $f: D \rightarrow \mathbb{R}$ is lower semicontinuous if $\liminf _{x \rightarrow x_{0}} f(x) \geq f\left(x_{0}\right)$ for each point $x_{0}$ in its domain $D$.

\section{RESET CONTROL DESIGN}

In this section we describe the motion system and friction characteristic, and then the design of the reset control law.

Consider a single-degree-of-freedom mass $m$ sliding on a horizontal plane with position $z_{1}$ and velocity $z_{2}$. The goal is to control the mass to the constant setpoint $\left(z_{1}, z_{2}\right)=(r, 0)$. The mass is subject to a control input $\bar{u}$ and a friction force taking values according to the set-valued mapping of the velocity $z_{2} \rightrightarrows \Psi\left(z_{2}\right)$, as in the dynamics:

$$
\begin{aligned}
& \dot{z}_{1}=z_{2}, \\
& \dot{z}_{2} \in \frac{1}{m}\left(\Psi\left(z_{2}\right)+\bar{u}\right) .
\end{aligned}
$$

The friction characteristic $\Psi$ consists of Coulomb friction with unknown static friction $\bar{F}_{s}$, and a viscous contribution $\alpha z_{2}$, with $\alpha \geq 0$ the viscous friction coefficient, i.e.,

$$
\Psi\left(z_{2}\right):=-\bar{F}_{s} \operatorname{Sign}\left(z_{2}\right)-\alpha z_{2} .
$$

In this paper, we primarily focus on robust compensation of unknown Coulomb friction, and on transient performance improvement. We therefore assume that a velocity-weakening (Stribeck) effect is absent in $\Psi$ (in the presence of such an effect, a velocity-dependent compensation control term may be employed as in [10]). Let us now formulate the control problem addressed in this paper:

Problem 1. Design a reset PID controller for input $\bar{u}$ in (1) that 1) globally asymptotically stabilizes the setpoint $\left(z_{1}, z_{2}\right)=$ $(r, 0)$, for any constant $r$, robustly w.r.t. any unknown static friction $\bar{F}_{s}$, and 2$)$ improves the transient performance w.r.t. a classical PID controller.

An integral action in the control input $\bar{u}$ is applied because it is able to compensate for unknown static friction $\bar{F}_{s}$ (which is typically the case in industrial motion applications). Before presenting a reset PID controller, we first introduce the classical PID controller to generate $\bar{u}$ as:

$$
\begin{aligned}
\bar{u} & =-\bar{k}_{p}\left(z_{1}-r\right)-\bar{k}_{d} z_{2}-\bar{k}_{i} z_{3}, \\
\dot{z}_{3} & =z_{1}-r,
\end{aligned}
$$

where $\bar{k}_{p}, \bar{k}_{d}, \bar{k}_{i}>0$ represent the proportional, derivative and integral gain, respectively. We apply then the following definitions to obtain mass-normalized system dynamics that favor clarity in the analysis in the upcoming sections:

$$
k_{p}:=\frac{\bar{k}_{p}}{m}, \quad k_{d}:=\frac{\bar{k}_{d}+\alpha}{m}, \quad k_{i}:=\frac{\bar{k}_{i}}{m}, \quad F_{s}:=\frac{\bar{F}_{s}}{m} .
$$

By using (4), the resulting mass-normalized, closed-loop dynamics given by (1) and the classical PID in (3) satisfy

$$
\begin{aligned}
& \dot{z}_{1}=z_{2}, \\
& \dot{z}_{2} \in-F_{s} \operatorname{Sign}\left(z_{2}\right)-k_{p}\left(z_{1}-r\right)-k_{d} z_{2}-k_{i} z_{3}, \\
& \dot{z}_{3}=z_{1}-r,
\end{aligned}
$$

with the state vector $z=\left(z_{1}, z_{2}, z_{3}\right) \in \mathbb{R}^{3}$. We select the normalized controller gains so that they satisfy the following assumption.

Assumption 1. The control parameters $k_{p}, k_{d}, k_{i}$ satisfy $k_{i}>0, k_{p}>0, k_{p} k_{d}>k_{i}$.

When $F_{s}=0$ (a special, linear case of our setting), this assumption is equivalent, by the Routh-Hurwitz stability criterion, to ensuring global exponential stability of the equilibrium $z_{1}=r, z_{2}=z_{3}=0$ through a stabilizing PID controller. Assumption 1 is hence not restrictive.

In [9], it is proven that the set of equilibria

$$
\mathcal{A}:=\left\{z=\left(r, 0, z_{3}\right)|| z_{3} \mid \leq F_{s} / k_{i}\right\}
$$

of (5) is globally asymptotically stable under Assumption 1. However, the PID-controlled system (5) typically results in long settling times due to the depletion and refilling of the integral buffer that is required to overcome the static friction $F_{s}$ 


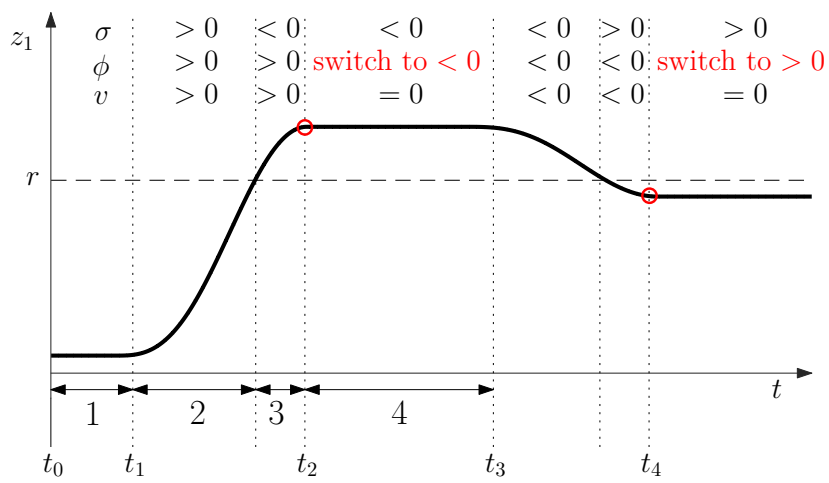

Fig. 1. Schematic representation of a position response with the proposed controller, including the sign of the states in $x$ per time interval. The red circles indicate the discrete jump instants.

upon overshoot, resulting in a change of sign of the integrator state of the PID (which is evident in the red dashed response of a classical PID in Fig. 3). This process is generally slow and takes increasingly more time with a decreasing position error, resulting in long periods of stick and thus a poor transient performance. Note that the system is said to be in a stick or slip phase when the state belongs respectively to the sets

$$
\begin{aligned}
\mathcal{E}_{\text {stick }} & :=\left\{z \in \mathbb{R}^{3}\left|z_{2}=0,\right| k_{i} z_{3}+k_{p}\left(z_{1}-r\right) \mid \leq F_{s}\right\} \\
\mathcal{E}_{\text {slip }} & :=\mathbb{R}^{3} \backslash \mathcal{E}_{\text {stick }} .
\end{aligned}
$$

In this paper, we propose then a novel reset PID control scheme to solve Problem 1. In particular, the proposed reset integral controller results in a significantly faster settling compared to the classical PID design in (3) (resulting in (5)), and does not suffer from the robustness issues of the switched PID controller presented in [10]. To this end, we replace the integrator in the PID controller (3) with a reset integrator. Only the integrator state is reset while keeping position and velocity unchanged, i.e.,

$$
z_{1}^{+}=z_{1}, z_{2}^{+}=z_{2}, z_{3}^{+}=-z_{3}-2 \frac{k_{p}}{k_{i}}\left(z_{1}-r\right),
$$

whenever $z=\left(z_{1}, z_{2}, z_{3}\right)$ is such that

$$
k_{p} k_{i}\left(z_{1}-r\right)^{2}+k_{i}^{2}\left(z_{1}-r\right) z_{3} \leq 0
$$

$$
\begin{array}{r}
\wedge-z_{2}\left(k_{p}\left(z_{1}-r\right)+k_{i} z_{3}\right) \leq 0 \\
\wedge\left|k_{p} k_{i}\left(z_{1}-r\right)^{2}+k_{i}^{2}\left(z_{1}-r\right) z_{3}\right| \geq \varepsilon
\end{array}
$$

for some design parameter $\varepsilon>0$, whose purpose is to avoid Zeno behaviour [17, p. 28-29]. The rationale behind the reset map for $z$ in (8a), the nontrivial reset conditions in (8b), and the role of $\varepsilon$ are clarified in Section III, where we show that, when the system enters a stick phase (see (7a)) after the position $z_{1}$ overshoots the setpoint $r$, conditions (8b) are satisfied. Moreover, we show in Section IV that the reset map for $z_{3}^{+}$preserves global asymptotic stability of the set of equilibria (6). In Section V, it is shown that the proposed reset integrator significantly improves transient performance, while the resets do not increase the risk of exciting high-frequency system dynamics, compared to the classical PID.

\section{HYBRID SYSTEM FORMULATION}

In this section, we rewrite the closed-loop reset control system (5), (8) in the hybrid systems formalism of [17], and discuss the rationale behind the proposed reset law (8).

Following [9], we introduce the coordinate transformation

$$
x:=\left[\begin{array}{c}
\sigma \\
\phi \\
v
\end{array}\right]:=\left[\begin{array}{c}
-k_{i}\left(z_{1}-r\right) \\
-k_{p}\left(z_{1}-r\right)-k_{i} z_{3} \\
z_{2}
\end{array}\right],
$$

where $\sigma$ is a generalized position error, $\phi$ is a generalized controller state encompassing the proportional and integrator control action, and $v$ is the velocity of the mass. This coordinate transformation simplifies the description of the system, transforms any constant setpoint $r$ to the setpoint 0 , facilitates the construction of a Lyapunov-like function for the stability analysis in Section IV, and rewrites the stick set in (7a) as

$$
\mathcal{E}_{\text {stick }}=\left\{x \in \mathbb{R}^{3}|v=0,| \phi \mid \leq F_{s}\right\} .
$$

The generalized controller state $\phi$ represents all the nonzero components of the control action at zero velocity (that is, the proportional and integral terms), and the difference between $\phi$ and the static friction $F_{s}$ at zero velocity determines then whether the system resides in a stick phase or not, see (10). 

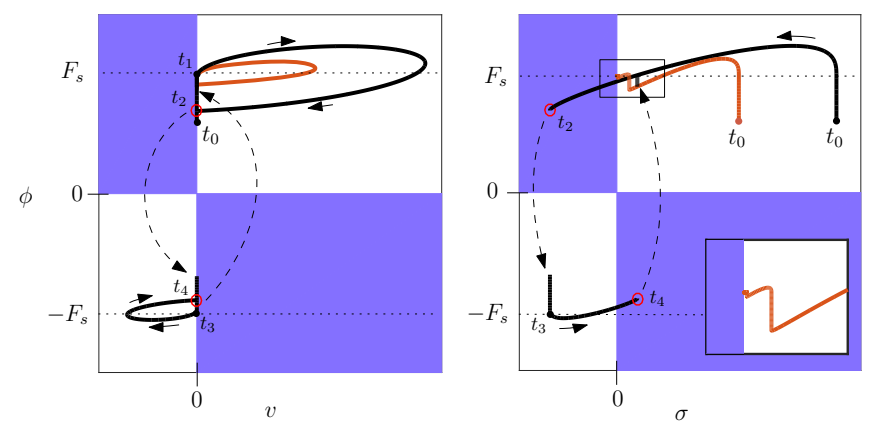

Fig. 2. Phase portraits corresponding to the response in Fig. 1 (black). The jump criteria $\phi v \leq 0$ (left) and $\phi \sigma \leq 0$ (right) are indicated in blue. A state jump (from the red circles) follows the dashed arrows. The red line corresponds to a different solution without overshoot, which then comes close to the jump set but never enters it, as designed (see the zoomed box). For clarity, we did not show the condition $|\phi \sigma| \geq \varepsilon$ in the figure. See Fig. 1 for times $t_{0}, \ldots, t_{4}$.

With the coordinate transformation (9), we rewrite the closed-loop dynamics (5) with the reset law (8) in the hybrid formalism of [17] as:

$$
\begin{aligned}
\dot{x} \in F(x):=\left[\begin{array}{c}
-k_{i} v \\
\sigma-k_{p} v \\
\phi-k_{d} v-F_{s} \operatorname{Sign}(v)
\end{array}\right], & x \in \mathcal{C}, \\
x^{+}=g(x):=\left[\begin{array}{lll}
\sigma & -\phi & v
\end{array}\right]^{\top}, & x \in \mathcal{D},
\end{aligned}
$$

where $F$ and $g$ are the flow and jump map. Resets are associated to the jump set

$$
\mathcal{D}:=\left\{x \in \mathbb{R}^{3}|\phi v \leq 0, \phi \sigma \leq 0,| \phi \sigma \mid \geq \varepsilon\right\},
$$

whereas the flow set is given by

$$
\mathcal{C}:=\overline{\mathbb{R}^{3} \backslash \mathcal{D}} .
$$

We argue now that the design of the jump set $\mathcal{D}$ improves the transient performance as per item 2) of Problem 1. Recall that we want the integrator to be reset (i.e., a jump in the hybrid formulation in (11)) when the system satisfies the following two conditions at the same time: 1) it enters a stick phase, and 2) the position overshoots the setpoint. Indeed, a reset in such conditions greatly reduces the time for the depletion and refilling of the integrator buffer, and consequently the stick duration. The transient performance is improved accordingly. Let us now illustrate this through a schematic response in Fig. 1 and 2.

1) Suppose the system has initial condition $z_{1}<r$ (and thus $\sigma>0$ ) and starts in a stick phase (time interval 1 in Fig. 1). Due to the dynamics of the integrator in (5), we have that $\phi>F_{s}$ will be reached, which results in a slip phase (intervals 2 and 3 in Fig. 1). The system enters a stick phase again (interval 4 in Fig. 1) when $v=0$ is reached and the controller state $\phi$ satisfies $0<\phi<F_{s}$, see the black response in Fig. 2. At this point, the condition $\phi v \leq 0$ is satisfied. A reset should not take place if the system enters a stick phase without the occurrence of overshoot, see the red response in Fig. 2. This may happen for different initial conditions, tuning, or friction characteristics, although item 1) (i.e., the system entering a stick phase) is satisfied in such situations. For this reason, we require a second condition $(\phi \sigma \leq 0)$ in the jump set $\mathcal{D}$ in $(11 \mathrm{c})$ :

2) Before an overshoot of the setpoint (interval 2 in Fig. 1), we have positive $\sigma$ and $\phi$, and thus $\phi \sigma>0$. After an overshoot (interval 3 in Fig. 1), $\sigma$ changes sign so that $\phi \sigma \leq 0$. Along with item 1), we conclude that the requirement $\phi \sigma \leq 0$ in $\mathcal{D}$ indeed enforces that a reset only takes place when the system enters a stick phase after an overshoot.

Finally, the condition $|\phi \sigma| \geq \varepsilon$ in (11c), for some design parameter $\varepsilon>0$, prevents a jump when $\sigma$ or $\phi$ are zero, so that Zeno behavior is avoided. Note that the jump set $\mathcal{D}$ in (11c) coincides with the reset conditions in (8b) for the original coordinates.

Remark 1. The criterion $\phi v \leq 0$ is chosen in the jump set $\mathcal{D}$ in (11c) rather than just $v=0$ to detect the stick phase, since the latter is hard to detect in practice due to velocity measurement noise. Although measurement noise around zero velocity may also render the product $\phi v$ sign indefinite due to chattering in the sign of $v$, the additional condition $\phi \sigma \leq 0$ in $\mathcal{D}$ prevents the system from experiencing undesired jumps. Indeed, after the first reset, the jump map (11b) ensures that $\phi \sigma>0$, thus $x \notin \mathcal{D}$.

Remark 2. The jump set $\mathcal{D}$ in (11c) is designed based on the new coordinates $x$. The states $\phi$ and $\sigma$ are unmeasurable in the case of an unknown mass $m$, as one can see from (9) and (4). However, even for an unknown $m$, we can define and obtain measurable states $\varsigma:=m \sigma=-\bar{k}_{i}\left(z_{1}-r\right)$ and $\varphi:=m \phi=-\bar{k}_{p}\left(z_{1}-r\right)-\bar{k}_{i} z_{3}$ from (9) and (4). This leads to jump conditions that can be checked based on measurable $\varsigma$ and $\varphi$ (note that for some $\epsilon>0,|\varphi \varsigma| \geq \epsilon$ can replace $|\phi \sigma| \geq \varepsilon$ since $\varepsilon$ is a design parameter). 


\section{STABiLity ANALYSis}

The set of equilibria (6) can be rewritten by the coordinate transformation in (9) as

$$
\mathcal{A}=\left\{x \in \mathbb{R}^{3}|\sigma=v=0,| \phi \mid \leq F_{s}\right\} .
$$

In this section, we show that (12) is globally asymptotically stable for (11), solving item 1) of Problem 1, as in the next Theorem.

Theorem 1. Under Assumption 1, $\mathcal{A}$ in (12) is globally asymptotically stable for (11).

The remainder of this section is devoted to outlining the main steps in the stability analysis that leads to the proof of Theorem 1. The proofs of these steps build upon the ones presented in [9], but are significantly challenged due to the addition of the reset control that gives rise to a hybrid (and no longer purely continuous-time) closed-loop system. Due to space restrictions, the proofs of the lemmas below are omitted and will be published elsewhere.

Consider the discontinuous Lyapunov-like function $V: \mathbb{R}^{3} \rightarrow \mathbb{R}$ defined as

$$
V(x):=\left[\begin{array}{c}
\sigma \\
v
\end{array}\right]^{\top}\left[\begin{array}{cc}
\frac{k_{d}}{k_{i}} & -1 \\
-1 & k_{p}
\end{array}\right]\left[\begin{array}{l}
\sigma \\
v
\end{array}\right]+\min _{F \in F_{s} \operatorname{Sign}(v)}(\phi-F)^{2} .
$$

We start by providing some properties of solutions while flowing, as in Lemma 1 below. To this end, we note that (11a) (and function (13)) suggests that during flow there are three relevant affine subsystems corresponding to the system being in slip with nonnegative or nonpositive velocity, and being in stick (cf. (7b) and (10)). With the definitions

$$
A:=\left[\begin{array}{ccc}
0 & 0 & -k_{i} \\
1 & 0 & -k_{p} \\
0 & 1 & -k_{d}
\end{array}\right], b:=\left[\begin{array}{c}
0 \\
0 \\
F_{s}
\end{array}\right], P:=\left[\begin{array}{ccc}
\frac{k_{d}}{k_{i}} & 0 & -1 \\
0 & 1 & 0 \\
-1 & 0 & k_{p}
\end{array}\right],
$$

these three subsystems correspond to

$$
\begin{array}{ll}
\dot{\xi}=f_{1}(\xi):=A \xi-b, & \xi\left(t_{0}\right)=\xi_{1}, \\
\dot{\xi}=f_{0}(\xi):=\left[\begin{array}{ccc}
0 & 0 & 0 \\
1 & 0 & 0 \\
0 & 0 & 0
\end{array}\right] \xi, & \xi\left(t_{0}\right)=\xi_{0}, \\
\dot{\xi}=f_{-1}(\xi):=A \xi+b, & \xi\left(t_{0}\right)=\xi_{-1} .
\end{array}
$$

For $\xi=\left(\xi_{\sigma}, \xi_{\phi}, \xi_{v}\right) \in \mathbb{R}^{3}$ and $|\xi|_{P}^{2}:=\xi^{T} P \xi$, define also

$$
V_{1}(\xi):=\left|\left[\begin{array}{c}
\xi_{\sigma} \\
\xi_{\phi}-F_{s} \\
\xi_{v}
\end{array}\right]\right|_{P}^{2}, V_{0}(\xi):=\left|\left[\begin{array}{c}
\xi_{\sigma} \\
0 \\
0
\end{array}\right]\right|_{P}^{2}, V_{-1}(\xi):=\left|\left[\begin{array}{c}
\xi_{\sigma} \\
\xi_{\phi}+F_{s} \\
\xi_{v}
\end{array}\right]\right|_{P}^{2}
$$

With these definitions in place, we can state Lemma 1 below. Item (i) asserts essentially that it is not possible to generate multiple solutions while they evolve in the flow set (in spite of the differential inclusion in (11a)) because they would coincide almost everywhere ${ }^{1}$. Item (ii) relates such a (unique) flowing solution with the solution of one of the subsystems (15a)-(15c). The hybrid time domain $\operatorname{dom} x$ of a solution $x$ to a hybrid dynamical system is defined in $[17, \S 2.2]$.

Lemma 1. For each solution $x$ to (11), each interval $I^{j}:=\{t:(t, j) \in \operatorname{dom} x\}=:\left[t_{j}, t_{j+1}\right]$ with nonempty interior, and for all $t \in\left(t_{j}, t_{j+1}\right)$,

(i) if $\hat{x}=(\hat{\sigma}, \hat{\phi}, \hat{v})$ is a solution to (11) on $\left[t, t^{\prime}\right) \times\{j\}$ with $t<t^{\prime} \leq t_{j+1}$ and $\hat{x}(t, j)=x(t, j)$, then $\hat{x}$ coincides with $x$ on $\left[t, t^{\prime}\right) \times\{j\}$;

(ii) one can select $k \in\{-1,0,1\}$ and $T>0$ such that the unique solution $\xi=\left(\xi_{\sigma}, \xi_{\phi}, \xi_{v}\right)$ to (15) with initial condition $\xi_{k}=x(t, j)$ coincides on $[t, t+T]$ with $x(\cdot, j)$, and additionally $V$ in (13) and $V_{k}$ in (15d) evaluated along $\xi$ satisfy for all $\tau \in[t, t+T]$ :

$$
V(\xi(\tau))=V_{k}(\xi(\tau)) \text { and } \frac{d}{d \tau} V_{k}(\xi(\tau)) \leq-c\left|\xi_{v}(\tau)\right|^{2},
$$

with

$$
c:=2\left(k_{p} k_{d}-k_{i}\right)>0 .
$$

Exploiting Lemma 1, we present the properties of $V$ in (13) in Lemma 2 below. Note that the distance of a point $x \in \mathbb{R}^{3}$ to the attractor $\mathcal{A}$ in (12) is obtained from the definition (by considering $\phi,-F_{s},|\phi| \leq F_{s}, \phi>F_{s}$ ) as

$$
|x|_{\mathcal{A}}^{2}:=\left(\inf _{y \in \mathcal{A}}|x-y|\right)^{2}=\sigma^{2}+v^{2}+\mathrm{dz}_{F_{s}}(\phi)^{2} .
$$

Lemma 2. $V$ in (13) is lower semicontinuous and enjoys the following properties:

1) $V(x)=0$ for all $x \in \mathcal{A}$ and there exists $c_{1}>0$ such that $c_{1}|x|_{\mathcal{A}}^{2} \leq V(x)$ for all $x \in \mathbb{R}^{3}$.

2) Each solution $x$ satisfies for $c$ in (17)

$$
V\left(x\left(t_{2}, j\right)\right)-V\left(x\left(t_{1}, j\right)\right) \leq-c \int_{t_{1}}^{t_{2}} v(t, j)^{2} d t,
$$

${ }^{1}\left[9\right.$, Lemma 1] proves uniqueness of solutions when $\mathcal{C}=\mathbb{R}^{3}, \mathcal{D}=\emptyset$. 
for all $t_{1}, t_{2}$ in each (flowing) interval $I^{j}:=\{t:(t, j) \in \operatorname{dom} x\}$ with nonempty interior, and $t_{1} \leq t_{2}$.

3) For all $x \in \mathcal{D}$ in (11c)

$$
V(g(x))-V(x) \leq 0 .
$$

The properties of $V$ in Lemma 2 imply that maximal solutions are complete [17, §2.3], as per the next lemma.

Lemma 3. For each initial condition $\bar{x} \in \mathbb{R}^{3}$, each maximal solution $x$ to (11) with $x(0,0)=\bar{x}$ is complete.

With these intermediate results, $\mathcal{A}$ can be proven to be globally attractive in Lemma 4 based on a meagre-limsup invariance principle [17, Thm. 8.11] (which applies since [17, Ass. 6.5] is satisfied by (11) and each maximal solution to (11) is complete by Lemma 3).

Lemma 4. $\mathcal{A}$ in (12) is globally attractive for dynamics (11).

We now turn to prove stability of $\mathcal{A}$ in (12). As in [9], we need the auxiliary function

$$
\hat{V}(x):=\frac{1}{2} k_{1} \sigma^{2}+\frac{1}{2} k_{2}\left(\mathrm{dz}_{F_{s}}(\phi)\right)^{2}+k_{3}|\sigma||v|+\frac{1}{2} k_{4} v^{2},
$$

in order to prove the stability bound (23) in Lemma 6 below for solutions traversing the directions of discontinuity of $V$ in (13). Indeed, because of such discontinuity on the attractor $\mathcal{A}$, an upper bound by $c_{2}|x|_{\mathcal{A}}^{2}$ for $V$ does not hold in $\mathbb{R}^{3}$, unlike the lower bound in Lemma 2 (item 1), and stability of $\mathcal{A}$ cannot be concluded directly from $V$. However, such lower and upper bounds, together with suitable growth bounds along solutions, can be established for $V$ and $\hat{V}$, respectively, in the following partition of the state space

$$
\begin{aligned}
& R:=\left\{x \mid v\left(\phi-\operatorname{sign}(v) F_{s}\right) \geq 0\right\}, \\
& \hat{R}:=\mathbb{R}^{3} \backslash R,
\end{aligned}
$$

as in the following lemma.

Lemma 5. For suitable positive scalars $k_{1}, k_{2}, k_{3}, k_{4}$ in (21), there exist positive scalars $c_{1}, c_{2}, \hat{c}_{1}, \hat{c}_{2}$ such that

$$
\begin{array}{ll}
c_{1}|x|_{\mathcal{A}}^{2} \leq V(x) \leq c_{2}|x|_{\mathcal{A}}^{2}, & \forall x \in R, \\
\hat{c}_{1}|x|_{\mathcal{A}}^{2} \leq \hat{V}(x) \leq \hat{c}_{2}|x|_{\mathcal{A}}^{2}, & \forall x \in \hat{R}, \\
\hat{V}^{\circ}(x):=\max _{\mathfrak{v} \in \partial \hat{V}(x), \mathfrak{f} \in F(x)}\langle\mathfrak{v}, \mathfrak{f}\rangle \leq 0, & \forall x \in \hat{R}, \\
\hat{V}(g(x))-\hat{V}(x)=0 & \forall x \in \hat{R},
\end{array}
$$

where $\partial \hat{V}(x)$ denotes the generalized gradient of $\hat{V}$ at $x$ as in [18, $\S 1.2], F$ is as in (11a), and $g$ is as in (11b).

The proof of (22a)-(22c) is carried out analogously to [9, Lemma 3], and (22d) holds since $\mathrm{dz}_{F_{s}}(\phi)^{2}=\mathrm{dz}_{F_{s}}(-\phi)^{2}$.

Finally, by composing the relations of Lemma 5 and Lemma 2 for $V$ and $\hat{V}$, the bound (23) of the following lemma can be obtained, which establishes (uniform global) stability (see [17, Def. 3.6]) of $\mathcal{A}$ in (12).

Lemma 6. Each solution $x$ to (11) satisfies

$$
|x(t, j)|_{\mathcal{A}} \leq \sqrt{\frac{c_{2} \hat{c}_{2}}{c_{1} \hat{c}_{1}}}|x(0,0)|_{\mathcal{A}},
$$

for all $(t, j) \in \operatorname{dom} x$.

Using Lemma 4 and 6, we conclude that $\mathcal{A}$ is globally asymptotically stable for hybrid dynamics (11). Note that, by the nonsingular coordinate transformation in (9), this is equivalent to item 1) of Problem 1).

\section{ILlustrative EXAMPLE}

In this section, we illustrate in a simulation study the transient performance improvements (item 2) of Problem 1) argued in Section III for the proposed reset controller. We use a numerical time-stepping scheme [19, Chap. 10] that is able to correctly deal with the set-valued friction characteristic $\Psi$.

Let $r=0$ be the constant position setpoint. First, consider only system (5), corresponding to a classical PID controller (3). The mass $m$ is unitary and the friction parameters are $\bar{F}_{s}=0.981 \mathrm{~N}$, and $\alpha=0.5$. We take $k_{p}=18, k_{d}=2.5$, and $k_{i}=40$, satisfying Assumption 1. The initial conditions are $z_{1}(0)=-0.05 \mathrm{~m}, z_{2}(0)=z_{3}(0)=0$, satisfying $z(0) \in \mathcal{E}_{\text {stick }}$ in (7a). The position response is visualized in Fig. 3a and $3 \mathrm{~b}$ in red, where the long periods of stick (resulting in long settling times) are evident. The control force $\bar{u}$ in (3) is presented in Fig. 3c, where the depletion and refilling process of the integrator buffer, causing long periods of stick, is clearly visible. This process takes increasingly more time with a decreasing position error, which results in increasingly longer periods of stick when the position error decreases.

Now consider (5) with the reset PID controller (8), whose resets correspond to $x \in \mathcal{D}$ as described in Section III. The position response is given in Fig. 3a and $3 \mathrm{~b}$ in blue, where the black circles indicate the instants of controller resets (which coincide with the start of a stick period). As it can also be observed in the control force $\bar{u}$ in Fig. $3 \mathrm{c}$, the reset mechanism 

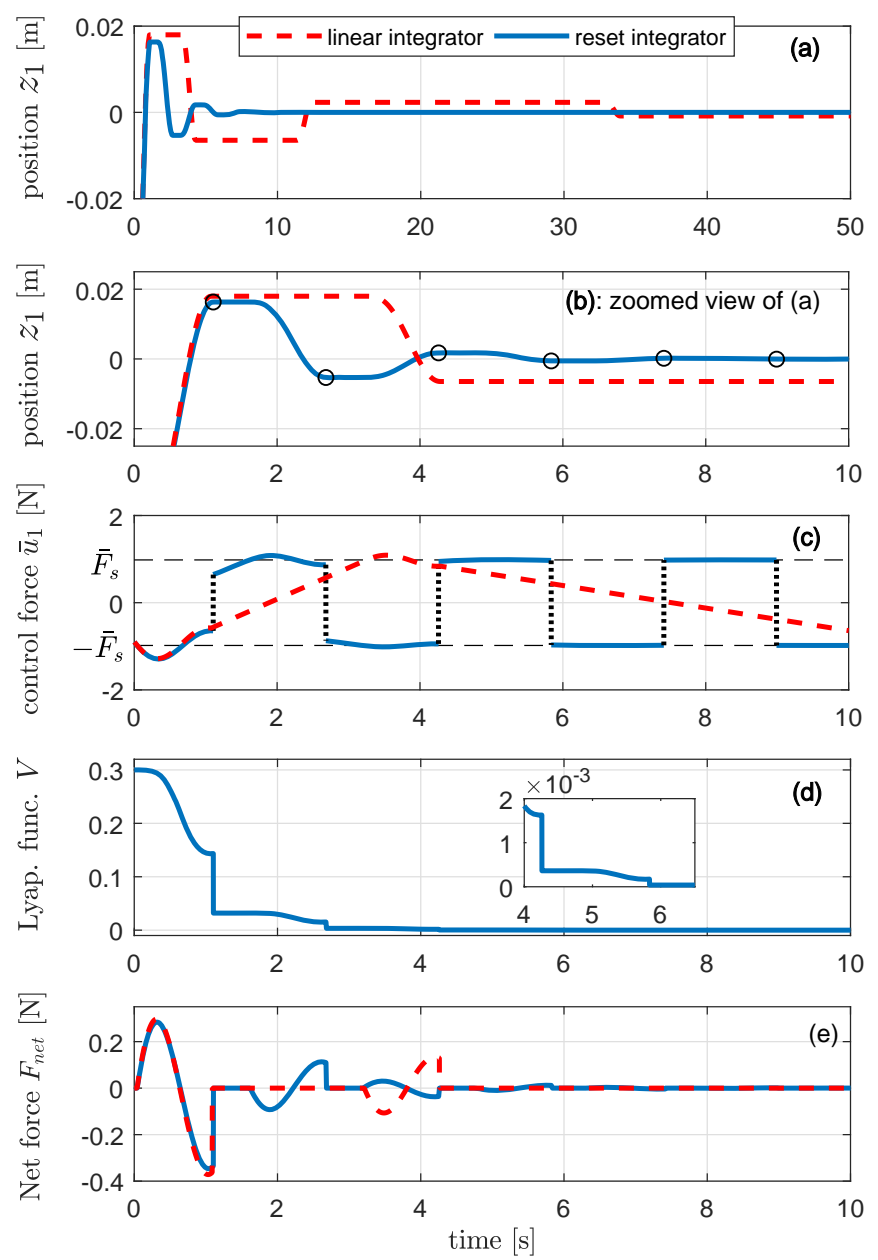

Fig. 3. Simulated position response with static and viscous friction (a,b), control force (c), evolution of the Lyapunov-like function (d), and net force acting on the system (e).

circumvents largely the depletion/refilling process of the integrator, decreasing significantly the settling time. When resets occur, the solution is in the stick set (10), namely $\bar{u}=m \phi$ with $|\phi| \leq F_{s}$, and $\bar{u}^{+}=-m \phi$ thanks to (11b). An increase or decrease in $\bar{u}$ is then visible after each reset since the integral action still needs to overcome the difference between $\phi$ and $F_{s}$ to exit the stick phase, so $|\bar{u}|$ converges to $\bar{F}_{s}$. In this sense, the control strategy identifies the unknown static friction level $\bar{F}_{s}$ in an online fashion.

Fig. 3d shows the evolution of the Lyapunov-like function (13). The discontinuities in $V$ evaluated along a solution are not due to the reset action, because the evaluated $V$ remains constant across jumps with $v=0$, as from (13) and (11b). Instead, the minimization term in (13) becomes zero instantaneously when solutions reach $\mathcal{E}_{\text {stick }}$ by flow.

Finally, we illustrate that the discontinuity in the control force caused by the proposed reset mechanism does not increase the risk of exciting high-frequency system dynamics, compared to the application of the classical PID controller. The essential insight is that, for the linear integrator, discontinuities in the net force are induced by discontinuities in the friction force, which are experienced only when the system enters the stick phase. We will argue in detail below that, also for the case of the reset integrator, discontinuities in the net force occur only when discontinuities in the friction force occur, which again are experienced only when the system enters the stick phase. Clearly, such discontinuities are inherent to the frictional nature of the system and are hence not induced by the hybrid nature of the controller.

Due to the fact that solutions to (11) are absolutely continuous during flow by definition [17, Def. 2.6] and that each solution $x$ cannot exhibit more than one consecutive jump (due to the definition of $g$ and $\mathcal{D}$ in (11)), solutions from $\mathcal{C}$ always enter $\mathcal{D}$ at a point at which $v=0$ (see also the left plot in Fig. 2). As a result, jumps only occur at zero velocity (except for the first jump if the initial conditions are chosen in the interior of $\mathcal{D}$ ). Given a state $x$, the net force $F_{n e t}(x)$ acting on the mass takes a value in the set $m \phi-\bar{F}_{s} \operatorname{Sign}(v)-\bar{k}_{d} v$. Consider the case of no controller reset and, correspondingly, a solution $x^{*}$ to only (11a) (with $\mathcal{C}=\mathbb{R}^{3}$ ) which experiences a slip-to-stick transition at time $t^{*}$. Also consider times $t_{-}^{*}$ and $t_{+}^{*}$ arbitrarily close to the left and right of $t^{*}$, respectively. At $t=t_{-}^{*}$, we have that $F_{n e t}\left(x^{*}\left(t_{-}^{*}\right)\right)=m \phi^{*}\left(t_{-}^{*}\right)-\bar{F}_{s} \operatorname{sign}\left(v^{*}\left(t_{-}^{*}\right)\right)-\bar{k}_{d} v^{*}\left(t_{-}^{*}\right) \neq 0$ indeed associated with a nonzero deceleration. At $t=t_{+}^{*}$, we have $F_{n e t}\left(x^{*}\left(t_{+}^{*}\right)\right) \in m \phi\left(t_{+}^{*}\right)-\bar{F}_{s} \operatorname{Sign}(0)$. We have $v^{*}\left(t_{+}^{*}\right)=0$ and $\left|\phi^{*}\left(t_{+}^{*}\right)\right| \leq F_{s}$ since $x^{*}\left(t_{+}^{*}\right) \in \mathcal{E}_{\text {stick }}$, and thus $m\left|\phi^{*}\left(t_{+}^{*}\right)\right| \leq \bar{F}_{s}$. It then follows from the combination of the system dynamics and the set-valued friction force law that the actual friction force (taken from the set $\bar{F}_{s} \operatorname{Sign}(0)$ ) equals 
$-m \phi^{*}\left(t_{+}^{*}\right)$ and thus $F_{n e t}\left(x^{*}\left(t_{+}^{*}\right)\right)=0$. The discontinuity in $F_{n e t}$ thus appears as a result of the set-valued friction force law, and is not caused by controller resets. Indeed, the discontinuity also appears in the case of the classical PID controller.

Therefore, the proposed hybrid PID controller achieves a significant transient performance improvement w.r.t. a classical PID controller, while not increasing the risk of exciting high-frequency system dynamics.

\section{CONCLUSION}

We have presented a reset integrator control strategy for motion systems with friction that achieves, firstly, robust global asymptotic stability of the setpoint for unknown static friction and, secondly, improves transient performance by reducing the settling time. Global asymptotic stability follows from a generalized invariance principle for hybrid dynamical systems. A numerical example shows the improved convergence speed of the proposed reset integrator, compared to a linear integrator. Future work encompasses experimental validation of the proposed reset controller, while also investigating the impact of microscopic frictional effects (e.g., stiffness-like characteristics) on the transient performance.

\section{REFERENCES}

[1] B. Armstrong-Hélouvry, P. Dupont, and C. Canudas de Wit, "A survey of models, analysis tools and compensation methods for the control of machines with friction," Automatica, vol. 30, no. 7, pp. 1083-1138, 1994.

[2] R. Hensen, M. van de Molengraft, and M. Steinbuch, "Friction induced hunting limit cycles: A comparison between the LuGre and switch friction model," Automatica, vol. 39, no. 12, pp. 2131-2137, 2003.

[3] D. Putra, H. Nijmeijer, and N. van de Wouw, "Analysis of undercompensation and overcompensation of friction in 1DOF mechanical systems," Automatica, vol. 43, no. 8, pp. 1387-1394, 2007.

[4] C. Makkar, G. Hu, W. G. Sawyer, and W. E. Dixon, "Lyapunov-based tracking control in the presence of uncertain nonlinear parameterizable friction," IEEE Trans. Autom. Control, vol. 52, no. 10, pp. 1988-1994, 2007.

[5] L. Freidovich, A. Robertsson, A. Shiriaev, and R. Johansson, "LuGre-model-based friction compensation," IEEE Trans. Control Syst. Technol., vol. 18, no. 1, pp. 194-200, 2010.

[6] D. Rijlaarsdam, P. Nuij, J. Schoukens, and M. Steinbuch, "Frequency domain based nonlinear feed forward control design for friction compensation," Mechanical Systems and Signal Processing, vol. 27, no. 1, pp. 551-562, 2012.

[7] N. van de Wouw and R. Leine, "Robust impulsive control of motion systems with uncertain friction," Int. J. of Robust and Nonlinear Control, vol. 22, pp. 369-397, 2012.

[8] J. Thomsen, "Using fast vibrations to quench friction-induced oscillations," Journal of Sound and Vibration, vol. 228 , no. 5, pp. 1079-1102, 1999.

[9] A. Bisoffi, M. Da Lio, A. R. Teel, and L. Zaccarian, "Global asymptotic stability of a PID control system with Coulomb friction," IEEE Trans. Autom. Control, 2018.

[10] R. Beerens, H. Nijmeijer, W. P. M. H. Heemels, and N. van de Wouw, "Set-point control of motion systems with uncertain set-valued Stribeck friction," in Proc. 20th IFAC World Congress, 2017.

[11] I. Horowitz and P. Rosenbaum, "Non-linear design for cost of feedback reduction in systems with large parameter uncertainty," International Journal of Control, vol. 21, no. 6, pp. 977-1001, 1975.

[12] O. Beker, C. Hollot, and Y. Chait, "Plant with integrator: an example of reset control overcoming limitations of linear feedback," IEEE Trans. Autom. Control, vol. 46, no. 11, pp. 1797-1799, 2001.

[13] W. Aangenent, G. Witvoet, W. Heemels, M. van De Molengraft, and M. Steinbuch, "Performance analysis of reset control systems," Int. J. of Robust and Nonlinear Control, vol. 20, no. 11, pp. 1213-1233, 2010.

[14] D. Nešić, A. Teel, and L. Zaccarian, "Stability and performance of SISO control systems with first-order reset elements," IEEE Trans. Autom. Control, vol. 56, no. 11, pp. 2567-2582, 2011.

[15] C. Prieur, S. Tarbouriech, and L. Zaccarian, "Lyapunov-based hybrid loops for stability and performance of continuous-time control systems," Automatica, vol. 49, no. 2, pp. 577-584, 2013.

[16] S. van Loon, K. Gruntjens, M. Heertjes, N. van de Wouw, and W. Heemels, "Frequency-domain tools for stability analysis of reset control systems," Automatica, vol. 82, pp. 101-108, 2017.

[17] R. Goebel, R. G. Sanfelice, and A. R. Teel, Hybrid Dynamical Systems: modeling, stability, and robustness. Princeton University Press, 2012.

[18] F. H. Clarke, Optimization and nonsmooth analysis. SIAM, 1990.

[19] V. Acary and B. Brogliato, Numerical Methods for Nonsmooth Dynamical Systems. Springer Science \& Business Media, 2008. 